\title{
The Effect of Parental Phubbing on Teenager's Mobile Phone Dependency Behaviors: The Mediation Role of Subjective Norm and Dependency Intention
}

This article was published in the following Dove Press journal:

Psychology Research and Behavior Management

\author{
Ru-De Liu' \\ Jia Wang ${ }^{2}$ \\ Dian $\mathrm{Gu}^{3}$ \\ Yi Ding $\mathbb{D}^{4}$ \\ Tian Po Oei $\mathbb{D}^{5,6}$ \\ Wei Hong ${ }^{3}$ \\ Rui Zhen ${ }^{7}$ \\ Yu-Meng $\mathrm{Li} \mathbb{B}^{3}$
}

'Beijing Key Laboratory of Applied Experimental Psychology, National Demonstration Center for Experimental Psychology Education, Faculty of Psychology, Beijing Normal University, Beijing, People's Republic of China; ${ }^{2}$ Teachers' College, Beijing Union University, Beijing, People's Republic of China; ${ }^{3}$ Faculty of Psychology, Beijing Normal University, Beijing, People's Republic of China; ${ }^{4}$ Division of Psychological and Educational Services, Graduate School of Education, Fordham University, New York, NY, USA; ${ }^{5}$ School of Psychology, University of Queensland, St Lucia, QLD 4072, Australia;

${ }^{6}$ Department of Psychology, James Cook University, 387380, Singapore; ${ }^{7}$ Institute of Psychological Sciences, College of Education, Hangzhou Normal University, Hangzhou, People's Republic of China

Correspondence: Jia Wang Teachers' College, Beijing Union University, Beijing I000II, People's Republic of China

Email wangjia@mail.bnu.edu.cn
Purpose: The aims of this study were to examine (a) the effects of parental phubbing on teenagers' mobile phone dependency and (b) the mediating roles of subjective norm and dependent intention of underlying this relationship.

Methods: We recruited 605 middle school students in Beijing, China and they completed the parental phubbing behaviors, subjective norm, dependency intention, and mobile phone dependency behavior questionnaires.

Results: The results of the structure equation modeling revealed that parental phubbing behaviors significantly increased teenager's mobile phone dependency behaviors in two indirect ways. First, parental phubbing reinforced teenagers' mobile phone dependency intention, which in turn increased the likelihood of mobile phone dependency. Second, parental phubbing enhanced the tendency of parental mobile phone dependence norm perceived by teenagers, and thus reinforced their mobile phone dependency intention, ultimately increasing mobile phone dependency.

Conclusion: We concluded that parental phubbing is a significant indicator of teenager mobile phone dependency and that mobile phone dependency intention plays a mediation role between them. In addition, the perceived parental mobile phone dependency norm played a mediation role between parental phubbing and mobile phone dependency intention and indirectly influenced the level of mobile phone dependency behaviors through the mediation effect of mobile phone dependency intention.

Keywords: parental phubbing, mobile phone dependency, dependency intention, subjective norm

With the rapid development of technology and science, mobile phone use has become an inseparable part of people's daily lives all over the world. According to the 40th Statistical Report on Internet Development of China Internet Network Center, ${ }^{1}$ the number of China's netizens in 2016 reached 724 million, of which $19.4 \%$ were from 10 to 19 years old and $24.8 \%$ were students ranged from elementary school to high school. In an investigation of mobile phone dependency among 3952 middle school students from 10 street blocks in Shenzhen, China, 50\% of the students had middle-level dependency and $22 \%$ had high-level dependency. ${ }^{2}$

Mobile phone dependency refers to the overuse of mobile phones, ${ }^{3,4}$ which can lead to physical and mental problems such as anxiety and fear of no mobile phone access. ${ }^{5-9}$ It is also known as problematic or excessive mobile phone use in some studies. ${ }^{10-12}$ Although mobile phone dependency may result in addictive behaviors which might be 
similar to those associated with substance abuse, ${ }^{13,14}$ they are not identical terms, because the core symptoms of mobile phone dependency are different from addiction. ${ }^{11,12}$ According to previous studies, high-level MP dependency is associated with physical complaints such as insomnia, headache, and fatigue ${ }^{15}$ mental disorders such as depression; and impaired social interactions and loneliness. ${ }^{9,16-18}$ Therefore, students' MP dependency can have a negative impact on students' behaviors, such as negative social relationships and low academic performance. ${ }^{19-21}$

Numerous studies have explored the topic of students' MP dependency and the environmental and psychological factors contributing to such dependency. Some of these studies focused on the effects of individual traits or other self-related factors. For example, Hong et al (2012) found that social extraversion and anxiety increased problematic MP use and self-esteem decreased problematic MP use. ${ }^{22}$ Jin et al (2017) demonstrated that the variable of attachment style can predict students' MP dependency with the mediation effect of self-esteem and dysfunctional attitudes. ${ }^{23}$ Likewise, Azam and Lubna (2013) proposed that subjective norm, which influences an individual's MP dependency intention, is an important factor predicting problematic MP use. ${ }^{24}$ Other scholars tested the impact of social environment. Choi and Kim (2016) found that teenagers' problematic behaviors resulting from MP dependency were closely related to their parents' inconsistent attitudes. ${ }^{25}$ Walsh et al (2011) investigation among young Australians revealed that their MP behaviors were partially shaped by the in-group norms they were exposed to. ${ }^{26}$ Yang and Chuan-Lai (2017) concluded that college students who received the advice about limiting use of MP from parents and peers showed lower levels of MP dependency. ${ }^{27}$ In addition, several researchers attempted to integrate the effects of external and internal factors on MP dependency behaviors in a single study. Hall et al (2014) found that individuals' internal norm, their partner's norm, and their relationships with partners were all important factors in MP dependency. ${ }^{28} \mathrm{Kim}$ and Shin (2016) reported that social needs and the influence of society had positive influences on the intention and behaviors of MP dependency. ${ }^{29}$

To identify additional related factors associated with teenagers' MP dependency, this study aimed to explore parental phubbing, subjective norm, behavioral intention, and the relationships among these variables in order to provide a theoretical framework and implications for future research.

\section{Parental Phubbing Behaviors and MP Dependency}

Phubbing, a composite of "phone" and "snubbing" proposed in the Macquarie Dictionary of Australian English, refers to the social phenomenon of looking at a mobile device while interacting with others, which ultimately results in a disturbance of conversation and communication. ${ }^{30}$ This phubbing behavior has been observed in various demographic groups, including young adults ${ }^{31,32}$ and adults. ${ }^{33}$ Checking a mobile phone while engaged in other activities has become a common phenomenon in families and schools. ${ }^{34-36}$ Phubbing has caused increasing public concern and has promoted the implementation of projects such as UP2U ("Program for raising awareness of phubbing problem in public") which is a program that encourages people to put down their mobile phones and build more constructive interpersonal communications. ${ }^{37}$

From the perspective of social learning theory, ${ }^{38}$ parental phubbing has an impact on teenagers' MP dependency behaviors. Bandura's (1977) social learning theory emphasized that all individuals experience the effects of social environments. Family, especially parents, are an irreplaceable part of a child's life and exert a considerable influence on perceived social surroundings, inner cognition, and daily behaviors. ${ }^{38}$ Taylor (2010) highlighted that most students in middle school experience a vulnerable period in their lives during which they are prone to develop maladaptive habits due to lack of insight regarding the potential risks and consequences of those behaviors. ${ }^{39}$ They might model parents' maladaptive behaviors, such as excessive smoking and drinking, especially if they view those behaviors as normal.

Although previous studies have not examined the relation between parental phubbing and MP dependency, some studies provided evidence for an association between the two variables. Researchers have demonstrated that parents' behaviors play an important modeling role in the process of teenager's habit formation, especially for some maladaptive behaviors such as excessive alcohol use, drug use, ${ }^{40-44}$ smoking, ${ }^{45-48}$ gambling, ${ }^{49,50}$ and so on. That is to say, exposure to family environments that include parents' maladaptive behaviors may influence teenagers' attitudes towards and perceived risks associated with these maladaptive behaviors. Thus, they may be more vulnerable and prone to develop these maladaptive behaviors as compared with other teenagers whose parents do not engage in these behaviors. Similarly, parents' engagement in phubbing behavior may influence teenagers' MP dependency behavior and may be a predictive variable of teenagers' MP 
dependency levels. However, this proposition calls for further empirical investigation.

\section{Subjective Norm, Intention, and MP Dependency}

Even when people understand that MP dependency may lead to negative outcomes, it is difficult to stop engaging in them. The theory of planned behavior (TPB) proposed by Ajzen (1980) provides a plausible explanation. ${ }^{51}$ This theory has been widely used to explain a variety of maladaptive habits and related interventions. ${ }^{52-54}$ TPB states that an individual's behaviors are closely associated with behavioral intention and three predictors (attitude, perceived behavioral control, and subjective norm). Behavioral intention is an antecedent of an actual behavior. Attitude is an individual's positive or negative evaluation of a behavior. Perceived behavioral control is an individual's perceived efficacy about performing the target behavior. Subjective norm is an individual's behavior norm or belief shaped by his or her social surroundings such as friends, parents, or important others. In plain language, the concept of social norm means, "What do you think others would like you to do?" and acts as the motivation for conforming to this norm. Ajzen (1980) contended that attitude, perceived behavior control, and subjective norm have an impact on behavioral intention and can be used to predict subsequent behavior. ${ }^{51}$ By definition, among these three key predictors of behavior intention in TPB, subjective norm might have the strongest association with parental phubbing, because the parents are the most important others whose behaviors will influence their children's subjective norms. Furthermore, some studies have found that subjective norm, among the TPB factors, has the strongest association with MP use (i.e., sending and reading text messages while walking; using mobile social networking apps). ${ }^{55,56}$ For these reasons, this study focused on subjective norm as one predictor of MP dependency and proposed that subjective norm and behavioral intention are linked with individuals' MP dependency behaviors.

Some of the existing studies that applied TPB to problematic behavior demonstrated the predictive power of subjective norm and behavioral intention on problematic use of Internet. Pelling and White (2009) applied TPB to the use of social networking web sites (SNWs) and found that attitudes and norms were significant predictors of SNW intention and problematic use tendency of $\mathrm{SNW}^{57}$ Researchers have examined problematic applications of Internet from the perspective of $\mathrm{TPB}$, such as online trading, ${ }^{58,59}$ online learning, ${ }^{60,61}$ and gaming. ${ }^{62}$ This study proposed that subjective norm and behavioral intention would have similar effects on MP dependency. Thus, subjective norm might influence individuals' MP dependency intention, which in turn might have an impact on their MP dependency behaviors.

\section{Parental Phubbing, Subjective Norm, and Intention}

The literature has shown the special role of important others in the growth of teenagers. For example, peer influences containing social modeling and perceived norms are positively related to adolescents' alcohol use. ${ }^{63}$ Similarly, we may conceptualize students' MP dependency behaviors from the perspective of a self-regulatory system. Parental phubbing behaviors function as an environmental context and set a standard norm for student's MP usage, gradually changing the student's subjective norm, which means that the individual student accepts a lower standard of MP use based on his self-judgment and stimulates more intense intention on MP dependency. If the parents do not realize the negative effects of phubbing, the phubbing environment might be reinforced consistently (suggesting that phubbing is common and acceptable behavior) for dependency intention, thus increasing the possibility of students' MP dependency. Based on these findings, this study hypothesizes that parental phubbing will influence the teenager's MP dependency with the mediation role of subjective norm and dependency intention.

Existing studies have demonstrated the effects of parental phubbing or other negative behaviors on an individual's cognition and performance of MP dependency. Chotpitayasunondh and Douglas (2016) examined the relationship between phubbing and perceived phubbing norm and found that this norm was affected by the frequencies of both phubbing and being phubbed. ${ }^{64}$ Lee (2003) demonstrated that imitating others influenced MP use intention and behaviors through the mediation effect of perceived subjective norm. ${ }^{65}$ Conversely, Xu et al (2012) studied parents' attitudes toward and supervision of teenagers' MP use and found that teenagers' MP dependency behaviors decreased when they perceived that their parents spent less time on their own MPs and exercised more restrictions on family MP use. ${ }^{66}$

\section{The Current Study}

The current study sought to demonstrate the predictive power of parental phubbing for teenagers' MP dependency and explore the relationship between parental phubbing 
and teenagers' MP dependency among Chinese middle school students. Even though some studies have partially demonstrated the application of TPB to MP dependency and the effect of phubbing on teenagers' MP behaviors, few studies have integrated these variables into one structural model. We aimed to examine the role of parental phubbing and explain the interactive effects of important variables on the formation of teenagers' MP dependency.

The following specific hypotheses were tested:

H1: Parental phubbing behaviors will be positively related to the level of teenagers' MP dependency.

H2: Subjective norm will influence teenagers' MP dependency intentions and then influence their MP dependency behaviors.

H3: Teenagers' dependency intention will play a mediation role between parental phubbing and teenagers' MP dependency.

H4: Parental dependency norm will play a mediation role between parental phubbing and teenager's dependency intention, and then affect MP dependency with the chain effect of intention.

To sum up, we assumed a structural model integrating $\mathrm{H} 1, \mathrm{H} 2, \mathrm{H} 3$, and H4, and we expected that the proposed model (see Figure 1) would have a good model fit.

\section{Method}

\section{Participants and Procedures}

In total, 605 (females $=294$, males $=274$, the rest did not report gender) students who attended a middle school in Beijing, China, volunteered to complete the questionnaires and provide feedback. This school was one of the ordinary middle school in Beijing and only enrolled students who had local household registration. The mean age of the participants was $15.09 \pm 2.89$ years. One of them completed less than half of the questions, and thus was not included in further data analysis. Of the participants, 153 were in Grade 7,105 in Grade 8, 163 in Grade 10, and 171 in Grade 11; the only student in Grade 12 (age 20) was removed from the data analysis. Most of their parent's education level were high school (mother $=19.8 \%$, father $=18.5 \%$ ), undergraduate (junior/regular college) (mother $=61.6 \%$, father $=54.4 \%$ ), or postgraduate (graduate/ Ph.D.) (mother $=13.5 \%$, father $=22.2 \%)$

Participants completed the questionnaires during class time without a time limit. The questionnaire contained 20 items in total, including one subjective norm item, three

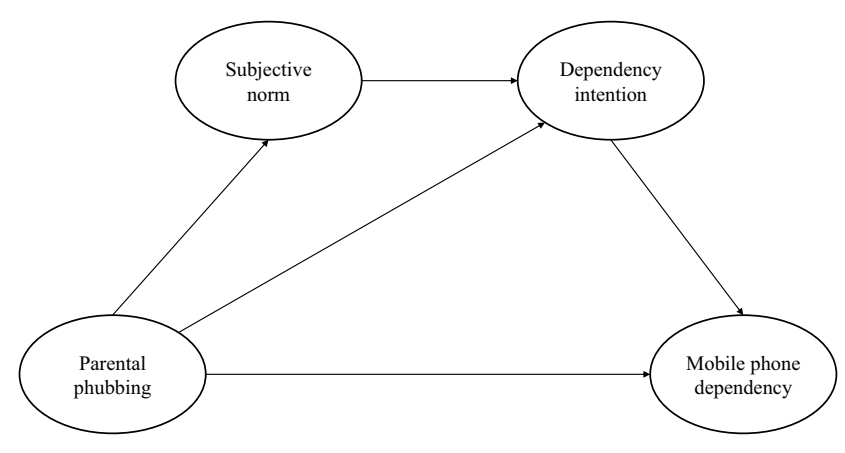

Figure I The hypothesized model.

dependency intention items, nine parental phubbing items, and seven MP dependency behavior items. The average time for completion was $10-15$ mins. There were minimal missing data and these were estimated with the maximum likelihood method. This study was approved by the Research Ethics Committee of Beijing Normal University and the principals of the participating schools. Written and Informed consent was obtained from all individual participants and from the parents/legal guardians of participants below the age of 16 . Parental written informed consent for participants between 16 and 18 was waived by the Research Ethics Committee of Beijing Normal University and the principals of the participating schools.

\section{Measures}

\section{Parental Phubbing Behaviors}

The scale was adapted from Partner Phubbing Scale ${ }^{67}$ and was used to measure teenagers' perceived parental phubbing. We changed the object of every statement into "my parents" and asked the participants to assess the frequency of each event on a 5-point Likert scale from never $=1$ to all the time $=5$ (i.e., When I had dinner with my parents, they checked their phones from time to time). The scale consisted of nine items, including one reverse wording item. The sum of the nine item scores represented the level of parental phubbing behaviors with higher scores represented more parental phubbing behaviors. Specifically, we conducted a CFA analysis with the nine-item construct. The CFA results showed that the nine items represented one dimension $\quad\left(\chi^{2}(36)=1583.042, \quad \mathrm{CFI}=0.919\right.$, TLI $=0.892$, RMSEA $(90 \% \mathrm{CI})=0.087[0.074,0.101])$ and had high reliability (alpha $=0.85$ ).

\section{Subjective Norm}

The scale of subjective norm measured the parent's latent attitudes toward children's MP dependency. The scale was adapted from $\mathrm{Lu}$ et al (2009). ${ }^{68}$ We translated the items 
under Subjective Norm dimension, noticeably, the original scale defined norm as users' perception of whether other important people perceive they should do this. However, the present study only focused on the influence of parents' negative behaviors on their children, so we changed the original words "people who are important to me" into "my parents" and measured teenagers' subjective norm under the influence of their parents. The statement of the item was worded as, "My parents think I don't have to control my time spent on the mobile phone." It was measured by a 5-point Likert scale from strongly disagree $=1$ to strongly agree $=5$. Higher reported scores represented higher levels of MP dependency norm from parents.

\section{Dependency Intention}

The dependency intention scale was adapted from $\mathrm{Lu}$ et al (2009). ${ }^{68}$ We translated the three items under Behavior Intention dimension and changed the original objection "instant messaging" into "time I spend on mobile phone" (i.e., I will recommend others to control the time spent on mobile phone). The items were measured by a 5-point Likert scale to assess participants' level of agreement with these statements, and one of them was a reversed item. A higher score represented the tendency to have a higher level of MP dependency intention. The alpha value (0.71) showed the stable reliability of the adapted scales.

\section{MP Dependency Behavior}

The measurement scale of teenager's MP dependency behavior was developed based on Dong et al's (2016) Mobile Dependency Scale $(\alpha=0.91){ }^{20}$ We used seven items in one dimension to measure MP dependency behavior. (i.e., the amount of time I spend using my cellular phone is increasing). Participants needed to make evaluations based on their personal experiences and then report the degree of agreement on a 4-point Likert scale from strongly no $=1$ to strongly yes $=4$. Higher scores represented higher levels of MP dependency behavior. The reliability of the scale (alpha $=0.88$ ) was relatively high.

\section{Analytical Strategy}

To calculate the correlation coefficients among variables and examine the effects of demographic variables (i.e., age and gender), we first used the SPSS 22.0 to conduct the correlation analysis. To prove the assumed relationship between parental phubbing behaviors and level of teenagers' MP dependency in $\mathrm{H} 1$ and the intervention effect of subjective norm and dependency intention stated in $\mathrm{H} 2$ and H3, we used path analysis with Mplus 7.0 to estimate the model fitting. In the chi-square test, the index reflected the deviation between the theoretical model and the actual observed model. The model is considered as having a good fit when the comparative fit index (CFI) was 0.90 or above. The value of root mean square error of approximation (RMSEA) was 0.08 or below, which is evidence for good model fit. ${ }^{69}$ In addition, to test the indirect effects, we used bootstrapping with 1000 samples to estimate the confidence intervals $(\mathrm{CI})$, which reflected the significance of effects by not including zero.

\section{Results}

\section{Common Method Variance Test}

Common method bias refers to the co-variation that is caused by identical data sources or raters, participants' similar testing environments or the contextual commonality of items or participants' similarity in characteristics, all of which can be categorized into system errors. This kind of bias causes confusion and serious misunderstanding of the analytical results. The current study could not exclude possible common method bias; therefore, we examined the latent constructs underlying all items using Harman's (1976) single factor test. ${ }^{70}$ The confirmatory factor analysis (CFA) extracted four common factors. The most contributory factor accounted for $29.7 \%$ of the co-variation for the entirety. The value was lower than the threshold value of $40 \%$. Therefore, we were assured that the participants in the present study completed the questionnaires independently. The analytical results did not have serious bias.

\section{Descriptive Statistics}

Descriptive statistics and correlations among variables are presented in Table 1, providing the basic data information. According to the descriptive statistics, gender had no effect on any of the other variables. Age showed a positive relation with dependency intention and MP dependency behaviors, which means that older teenagers are likely to show higher levels of mobile phone dependency intention at the present and in the future and engage in more MP dependency behaviors. In addition, four variables were significantly correlated. Specifically, parental phubbing was positively related to subjective norm, intention, and MP dependency behaviors, while MP dependency behaviors were positively related to the above three constructs. 
Table I Descriptive Statistics and Inter-Correlations of Variables in the Current Study $(N=602)$

\begin{tabular}{|c|c|c|c|c|c|c|c|}
\hline & $\mathrm{M} \pm \mathrm{SD}$ & $\mathbf{I}$ & 2 & 3 & 4 & 5 & 6 \\
\hline I. Gender & - & - & & & & & \\
\hline 2. Age & $16.09 \pm 2.89$ & 0.05 & - & & & & \\
\hline 3. Parental phubbing & $2.54 \pm 0.79$ & 0.02 & -0.04 & - & & & \\
\hline 4. Subjective norm & $1.73 \pm 1.10$ & -0.04 & 0.07 & $0.16^{* * *}$ & - & & \\
\hline 5. Dependency intention & $2.64 \pm 0.78$ & -0.06 & $0.15^{* * *}$ & $0.14^{* *}$ & $0.26 * * *$ & - & \\
\hline 6. Mobile phone dependency & $2.04 \pm 0.69$ & 0.06 & $0.09 *$ & $0.30^{* * *}$ & $0.22^{* * *}$ & $0.35 * * *$ & - \\
\hline
\end{tabular}

Notes: Gender was coded as I=male, $2=$ female. $* P<0.05 * * P<0.01 * * * P<0.001$

\section{Model Fitting Test}

We used Mplus 7.0 to conduct path analysis to test the hypothesized relationships among the four variables in Figure 1. Firstly, maximum likelihood (ML) estimation was conducted in terms of individual item factor loadings, the reliability of measures, convergent validity, and discriminant validity. Given that the variable of age had a potential influence on teenager's dependency intention and MP dependency (see Table 1), we controlled the deviation of age on these two variables. The results of the latent SEM showed that the data fitted the model well $\left(\chi^{2}(184)=2.968, \mathrm{CFI}=0.915\right.$, TLI $=0.903$, RMSEA $(90 \%$ $\mathrm{CI})=0.058[0.053,0.064]$ ) (see Figure 2).

To illustrate, the model included one direct path and two indirect paths. The direct path - from parental phubbing behaviors to teenagers' MP dependency behaviors - showed that parental phubbing increased the level of MP dependency $(\beta=0.171, p<0.01,95 \%$ CI $[0.038,0.167])$, which supported H1 that parental phubbing was positively related to teenagers' MP dependency behaviors.

There were also two indirect paths from parental phubbing to MP dependency: (a) through dependency intention and (b) mediated by subjective norm, via dependency intention, then to teenagers' MP dependency behaviors. The results also demonstrated that parental phubbing functioned

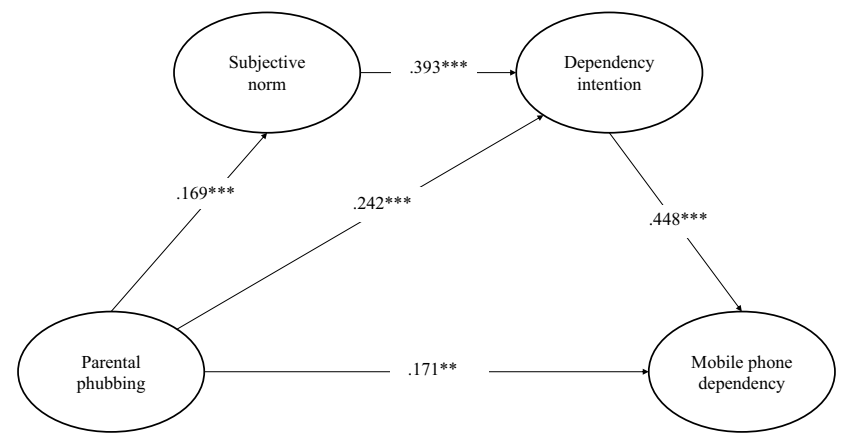

Figure 2 Mediation model of subjective norm and dependency intention between parental phubbing and teenager's mobile phone dependency. $* * p<0.01$; $* * *<0.001$. via path (a) and path (b). Specifically, in path (a), parental phubbing increased teenagers' MP dependency intention $(\beta=0.242, p<0.001,95 \%$ CI $[0.101,0.345])$ and then positively affected teenagers' MP dependency behaviors $(\beta=0.448, \mathrm{p}<0.001,95 \%$ CI [0.220, 0.417]). We also implemented Bootstrap $(=1000)$ test to examine the mediation effect, and the results showed the indirect effect of path (a) $(\beta=0.109, p<0.05,95 \%$ CI $[0.043,0.174])$, which demonstrated the mediation role of teenager's mobile phone intention between parental phubbing and teenagers' MP dependency (H3). In path (b), parental phubbing positively affected teenagers' subjective norm perceived from parents $(\beta=0.169, p<0.001,95 \%$ CI $[0.099,0.334])$, which in turn increased teenagers' MP dependency intention $(\beta=0.393$, $\mathrm{p}<0.001,95 \%$ CI $[0.205,0.350])$ and ultimately resulted in teenagers' higher-level MP dependency behaviors $(\beta=0.448$, $\mathrm{p}<0.001,95 \%$ CI $[0.220,0.417])$. According to the result of Bootstrap $(=1000)$ test, the indirect effect of path (b) $(\beta=0.030, p<0.05,95 \%$ CI $[0.010,0.050])$ testified the mediation role of subjective norm between parental phubbing and teenager's dependency intention, and the joint influence on MP dependency with the chain effect of intention was supported (H2, H4).

Meanwhile, the results also illustrated that the direct path parental phubbing to teenagers' MP dependency ( $\beta=0.171$, $\mathrm{p}<0.001,95 \%$ CI $[0.071,0.271])$ - accounted for $55.3 \%$ of the whole variation, and took up a greater proportion of than path (a) $35.3 \%(\beta=0.109, \mathrm{p}<0.05,95 \%$ CI $[0.043,0.174])$ and path (b) $9.7 \%(\beta=0.030, p<0.05,95 \%$ CI $[0.010,0.050])$

\section{Discussion}

The results of this study supported the four hypotheses. As proposed in H1, phubbing positively affected the level of MP dependency. More frequently, parental phubbing behaviors resulted in a higher level of MP dependency. Although no previous study on the same topic has been reported, the results of this study are consistent with some related studies. Ying (2012) showed that teenagers' 
perceived parental MP use could predict their own MP dependency ${ }^{71}$ concurring with the findings of the present study. Other scholars focused on the influence of parental monitoring on MP dependency and proposed that parental attitudes toward and monitoring of middle school students' internet or MP use decreased the likelihood of excessive use. ${ }^{72-74}$ This result can be explained by Bandura's social learning theory. ${ }^{75}$ Phubbing behaviors of parents serve as a model for MP use by middle school students. They will be likely to develop the phubbing behavior through the process of intending, performing, duplicating, and ultimately integrating the behavior into their daily activities through frequent interaction with their parents. Of note is that the influential role of peer relationships was not considered in the present study, ${ }^{35,76,77}$ because in most of the middle schools in China, students are not permitted to take MPs to school, which limits the opportunities to learn MP behavior in the context of peer experiences.

As proposed in $\mathrm{H} 2$, subjective norm and dependency intention could be influential factors in explaining the variation in teenagers' MP dependency. The finding is consistent with previous findings that applied TPB to online shopping, ${ }^{58}$ education, ${ }^{60}$ gaming, ${ }^{62}$ excessive social network use, ${ }^{57}$ and MP use in different scenarios (i.e., mobile phone use while crossing the road, cycling and driving). ${ }^{78-80}$ Liao et al (2007) studied continued use of e-services and found that subjective norm affected behavioral intention, which in turn affected actual behaviors. ${ }^{81}$ Piazza et al (2019) found that subjective norm significantly predicted the intention to use mobile phone while crossing the street. ${ }^{79}$ Jiang et al (2019) and Holland and Rathod (2013) reported the significant effect of subjective norm on MP use while cycling and driving, respectively. ${ }^{78,82}$ However, other scholars have reported different results. Fang and Shao (2007) explained individual's intention to participate in web-based surveys by using an extended TPB model and found that subjective norm did not significantly predict potential respondents' intention. ${ }^{83}$ In the current study, we focused on the effects of perceived subjective norm from parents because the concept had a close relationship with parental phubbing. Based on the definition of subjective norm, the construct is more closely associated with individuals' external guidance than it is with attitudes. Parental phubbing, as an important external factor, might have an immediate impact on an individual teenager. Finally, the results showed that perceived subjective norm from parents influenced teenagers' MP dependency intention and ultimately influenced their MP dependency behaviors. The present study focused on explaining the effects of subjective norm and intention and partially proved the practical application of TPB to an examination of MP dependency.

Moreover, the mediation role of MP dependency intention between parental phubbing behaviors and students' MP dependency behaviors (H3) was also supported by the indirective effect test of path (a). This result showed that parental phubbing could not only increase the likelihood of students' MP behaviors directly but could also increase the student's MP dependency intention and then impacted on higher-level MP behaviors. Although the previous studies could not fully support the present results, some studies did partially support that parents' maladaptive behavior such as excessive phubbing leads to higher-level MP use intention and behaviors. For example, Roberts and David (2016) demonstrated that phubbing was distractive for romantic partners' communication and that the ever-increasing use of cell phones provided an environment of drawing teenager's attention to imitate. ${ }^{67}$ Lee (2003) demonstrated that imitation of others influenced MP use intention. ${ }^{65}$

Additionally, the indirective effect test of path (b) supported the mediation effect of subjective norm and dependency intention in the relationship of parental phubbing and students' MP dependency behaviors. This is consistent with Bandura's self-efficacy theory (1977). ${ }^{38}$ On the one hand, people tend to look for vicarious experiences from important individuals when they lack related experiences or knowledge (Bandura, 1977) ${ }^{38}$ Therefore, when adolescents want to know a typical way to use MPs, parents' phubbing behaviors may be a vicarious experience for their children and may shape a biased cognition of MP use in adolescents' minds, which is equal to the subjective norm- the criterion for judging the usage of MPs. Thus, with the increase of parental phubbing behaviors, subjective norm becomes inclined to MP dependency. On the other hand, people might receive vicarious reinforcement from others (Bandura, 1977). ${ }^{38}$ Therefore, some other impacts caused by parental phubbing such as reducing communication anxiety will work out as a kind of reinforcement for adolescents. Then, they develop phubbing as an effective way to solve similar problems and receive more self-reinforcement in the process of phubbing replication, which increases dependency intention and induces more MP dependency behaviors. This chain effect showed similar results to some previous studies. For example, Chotpitayasunondh and Douglas (2016) demonstrated that an individual's phubbing is related to his or her 
frequency of phubbing behaviors, which represents the degree of self-reinforcement, and being phubbed, which represents the degree of vicarious reinforcement from others. ${ }^{64}$ Therefore, parents' phubbing behaviors not only contribute to a negative subjective norm for children but also serve as a reinforcement condition for teenagers' dependency intention which leads to teenagers' more MP dependency behaviors.

\section{Innovations and Limitations}

We believe our study was the first study of its kind to show that parents' phubbing behaviors can set a negative example in MP use for middle school students. This finding offered new insight for intervention strategies that control or limit parents' phubbing behaviors in the family environment, especially when parents are communicating with teenagers. Thus, parents can intervene in teenagers' phubbing intention and reduce teenagers' MP dependency behaviors. The effectiveness of this method should be tested in future experiments. In addition, the present study partially supported the validation and practicability of TPB when applying it to MP dependency. Previous studies have used this theory as a framework to investigate the formation of different behaviors, but few studies have validated the theory for MP dependency. Finally, our study contributed to the literature by providing an integrated model of MP dependency. We proposed a model to explain the process from parents' behaviors, via the influence of teenagers' perceptions and cognition, toward the final consequence of behaviors, showing more convincing and detailed evidence for the complicated relationships among the variables.

Although we tried to standardize all of the procedures and control for the influence of uncertain variables in this study as carefully as possible, some limitations were unavoidable. First, this study explored the mediating role of subjective norm, as one of the three determinants of behavior intention in TPB, between parental phubbing and MP dependency. However, TPB proposed that there are three determinants (attitude, perceived behavioral control, and subjective norm) that have association with an individual's behavior intention. ${ }^{51}$ It would be interesting for future researchers to investigate the effects of all of these three predictors of TPB on the relations of parental phubbing and MP dependency. Second, although the sample size of this study was 605 and was large enough to conduct structural equation modeling, our participants were from only one city in China, which limits the generalizability of the study. The findings might not be generalizable to all middle school students in other cities of China. Large regional disparities caused by socioeconomic status differences still exist among different areas of China. In addition, different schools have different guidelines for students' MP use. Future studies need to use a random sampling method with a larger sample size from more widespread areas to improve the generalizability of the results.

\section{Acknowledgments}

This study was supported by the National Social Science Foundation (grant number 17BSH102) to Ru-De Liu. Thanks to the parents, teachers, and students in participating schools. Thanks to the anonymous reviewers for their helpful and constructive comments.

\section{Compliance with Ethical Standards}

This study closely followed all ethical standards established by Institutional Review Board at the authors' universities and the participating schools.

\section{Disclosure}

The authors report no conflicts of interest in this work.

\section{References}

1. China Internet Network Information Center (CNNIC). The 41st Statistical Report on Internet Development in China. China: Cyberspace Administration of China; 2017.

2. Huang YY, Pei D, Zhou L.Analysis of mobile phone dependence of Shenzhen middle school students and its influencing factors. Chin Sch Health. 2017;38(9):1414-1416.

3. Billieux J. Problematic use of the mobile phone: a literature review and a pathways model. Curr Psychiatry Rev. 2012;8(4):299-307. doi: $10.2174 / 157340012803520522$

4. Xie SM, Yan MY. College students' "mobile phone dependence" problems and countermeasures. Jiangxi Educ. 2009;(z1):49-50. (Chinese).

5. Choliz M. Mobile-phone addiction in adolescence: the test of mobile phone dependence (TMD). Prog Health Sci. 2012;2(1):33-44.

6. Shi JG.Cell phone dependence syndrome. J Clin Psychiatry. 2009;19 (2):138-139.

7. Vacaru MA, Shepherd RM, Sheridan J. New Zealand youth and their relationships with mobile phone technology. Int J Mental Health Addict. 2014;12(5):572-584. doi:10.1007/s11469-014-9488-z

8. Wang J. Investigation and research on college students' mobile phone dependence. Sci Wealth. 2010;9:73-74.

9. Yen CF, Tang TC, Yen JY, et al. Symptoms of problematic cellular phone use, functional impairment and its association with depression among adolescents in southern Taiwan. $J$ Adolesc. 2009;32(4):863. doi:10.1016/j.adolescence.2008.10.006

10. Bianchi A, Phillips JG. Psychological predictors of problem mobile phone use. CyberPsychol Behav. 2005;8(1):39-51. doi:10.1089/ cpb.2005.8.39

11. Oviedo-Trespalacios O, Nandavar S, Newton JDA, Demant D, Phillips JG. Problematic use of mobile phones in Australia is it getting worse? Fronti Psychiatry. 2019;10:105. doi:10.3389/fpsyt.2019.00105

12. Tayana $\mathrm{P}$, Xavier C. Is smartphone addiction really an addiction? J Behav Addict. 2018;7(2):252-259. doi:10.1556/2006.7.2018.49 
13. Leung L. Linking psychological attributes to addiction and improper use of the mobile phone among adolescents in Hong Kong. J Children Media. 2008;2(2):93-113. doi:10.1080/17482790802078565

14. Park WK. Mobile phone addiction. In: Long R, Pedersen PE, editors. Mobile Communications: Re-Negotiation of the Social Spheres. London (UK): Springer; 2005:253-272.

15. Söderqvist F, Carlberg M, Hardell L. Use of wireless telephones and self-reported health symptoms: a population-based study among Swedish adolescents aged 15-19 years. Environ Health. 2008;7 (1):1-10. doi:10.1186/1476-069X-7-18

16. Jiang YZ, Bai XL.Influence of college student mobile internet dependence on alienation: the role of social support system. Psychol Dev Educ. 2014a;30(5):540-549.

17. Jiang YZ, Bai XL.The relationship between college students' mobile internet dependence and loneliness: the mediating role of social support on the Internet. Chin Spec Educ. 2014b;163(1):41-47.

18. Liu H, Wang HL. The relationship among university student's mobile addiction, mobile phone use motive and loneliness. Psychol Sci. 2011;34(6):1453-1457.

19. Amidtaher M, Saadatmand S, Moghadam Z, Fathi G, Afshar R. The relationship between mobile cell phone dependency, mental health and academic achievement. Am J Educ Res. 2016;4(5):408-411.

20. Dong GS, Park Y, Min KK, Park J. Mobile phone dependency and its impacts on adolescents' social and academic behaviors. Comput Human Behav. 2016;63:282-292. doi:10.1016/j.chb.2016.05.026

21. Qu X-Y, Lu A-T, Song P-F, Lan Y-L, Cai R-Y. The mechanism of mobile phone addiction influencing academic burnout with mediating effect of procrastination. Chin J Appl Psychol. 2017;23(1):49-57.

22. Hong FY, Chiu SI, Huang DH. A model of the relationship between psychological characteristics, mobile phone addiction and use of mobile phones by Taiwanese university female students. Comput Human Behav. 2012;28(6):2152-2159. doi:10.1016/j.chb.2012.06.020

23. Jin Y, Sun C, An J, Li J. Attachment styles and smartphone addiction in Chinese college students: the mediating roles of dysfunctional attitudes and self-esteem. Int J Mental Health Addict. 2017;15 (5):1122-1134. doi:10.1007/s11469-017-9772-9

24. Azam S, Lubna N. Mobile phone usage in Bangladesh: the effects of attitude towards behaviour and subjective norm. Annamalai Int J Bus Stud Res. 2013;5:25-34.

25. Choi K, Kim M. An effect of parenting attitude on dependency on mobile phone of youth and mediative effect of peer relationship and academic attitudes. Focused Gender Difference. 2016;48:109-133.

26. Walsh SP, White KM, Cox S, Young RM. Keeping in constant touch: the predictors of young Australians' mobile phone involvement. Comput Human Behav. 2011;27(1):333-342. doi:10.1016/j.chb.2010.08.011

27. Yang GJ, Chuan-Lai HU.Analysis on prevalence and influencing factors of mobile phone dependency among college students in Bengbu City. J Bengbu Med Coll. 2017;42(2):227-231.

28. Hall JA, Baym NK, Miltner KM. Put down that phone and talk to me: understanding the roles of mobile phone norm adherence and similarity in relationships. Mob Media Commun. 2014;2(2):134-153. doi:10.1177/2050157913517684

29. Kim DE, Shin JI. The impacts of dependency and addition of smartphone on behavioral intentions in South Korea. Adv Sci Technol Lett. 2016;126:107-111.

30. Bragazzi NL, Del PG. A proposal for including nomophobia in the new DSM-5. Psychol Res Behav Manage. 2014;7:155-160. doi:10.2147/PRBM.S41386

31. Karadağ E, Tosuntaş SB, Erzen E, et al. The virtual world's current addiction: phubbing. Addicta. 2016;3(2):250-269. doi:10.15805/ addicta.2016.3.0013

32. Wang ZY. Investigation and analysis of the phenomenon of "downcast clan" in university classrooms: taking the school of elementary education of Tianjin Normal University and the school of electronic science and technology of Tianjin Polytechnic University as an example. J Northwest Inst Educ. 2016;(6):61-63. (Chinese).
33. Wang X, Xie X, Wang Y, Wang P, Lei L. Partner phubbing and depression among married Chinese adults: the roles of relationship satisfaction and relationship length. Pers Indiv Dif. 2017;110:12-17. doi:10.1016/j.paid.2017.01.014

34. Lee SJ, Chae YG. Children's internet use in a family context: influence on family relationships and parental mediation. Cyberpsychol Behav. 2007;10(5):640-644. doi:10.1089/cpb.2007.9975

35. Lim MY, Yang M. The effects of parental child-rearing attitudes and peer relationships on children's mobile phone dependency and use type. Korean J Human Ecol. 2014;23(2):163-174. doi:10.5934/kjhe.2014.23.2.163

36. Shi X, Yang L.A research on the phubbing phenomenon in Hetao College. J Hetao Coll. 2016;13(2):55-58.

37. Metsiritrakul K, Puntavachirapan N, Kobchaisawat T, Leelhapantu S, Chalidabhongse TH (2016). UP2U: program for raising awareness of phubbing problem with stimulating social interaction in public using augmented reality and computer vision. 13th International Joint Conference on Computer Science and Software Engineering (JCSSE), Khon Kaen, Thailand. (pp. 1-6).

38. Bandura A. Self-efficacy: toward a unifying theory of behavioral change. Adv Behav Res Ther. 1977;1(4):139-161. doi:10.1016/01466402(78)90002-4

39. Taylor SE. Health Psychology. 7th ed. Boston (MA): McGraw-Hill Higher Education; 2010.

40. Black R, Mayer J. Parents with special problems: alcoholism and opiate addiction. Child Abuse Negl. 1980;4(1):45-54. doi:10.1016/ 0145-2134(80)90033-2

41. Bond GE, Burr RL, McCurry SM, Rice MM, Borenstein AR, Larson EB. Alcohol and cognitive performance: a longitudinal study of older Japanese Americans. Int Psychogeriatr. 2005;17 (4):653-668. doi:10.1017/S1041610205001651

42. Hansen WB, Graham JW, Sobel JL, Shelton DR, Flay BR, Johnson CA. The consistency of peer and parent influences on tobacco, alcohol, and marijuana use among young adolescents. J Behav Med. 1987;10(6):559-579. doi:10.1007/BF00846655

43. McKeganey N, Barnard M, McIntosh J. Paying the price for their parents' addiction: meeting the needs of the children of drug-using parents. Drugs Educ Prev Policy. 2001;9(3):233-246. doi:10.1080/09687630210122508

44. Puxi M. Preventing Addiction and Self-Help. Children with Alcoholand Drug-Addicted Parents - A Family Politics Model Project. DISKURS. 1999;9(2):30-39.

45. Abdullah AS, Mak YW, Loke AY, Lam TH. Smoking cessation intervention in parents of young children: a randomised controlled trial. Addiction. 2005;100(11):1731-1740. doi:10.1111/j.1360-0443.2005.01231.x

46. Strachan DP, Cook DG. Health effects of passive smoking v. parental smoking and allergic sensitization in children. Thorax. 1998;53 (2):117-123. doi:10.1136/thx.53.2.117

47. Tilson EC, McBride CM, Lipkus IM, Catalano RF. Testing the interaction between parent-child relationship factors and parent smoking to predict youth smoking. J Adolesc Health. 2004;35(3):182-189. doi:10.1016/S1054-139X(03)00532-9

48. Tyc VL, Klosky J, Throckmorton-Belzer L, Lensing S, Rai SN. Parent-reported environmental tobacco smoke exposure among preadolescents and adolescents treated for cancer. Psycho-Oncology. 2004;13(8):537-546. doi:10.1002/(ISSN)1099-1611

49. Langhinrichsen-Rohling J, Rohde P, Seeley JR, Rohling ML. Individual, family, and peer correlates of adolescent gambling. J Gambling Stud. 2004;20(1):23-46. doi:10.1023/B:JOGS.0000016702.69068.53

50. Vachon J, Vitaro F, Wanner B, Tremblay RE. Adolescent gambling: relationships with parent gambling and parenting practices. Psychol Addict Behav. 2004;18(4):398-401. doi:10.1037/0893-164X.18.4.398

51. Ajzen I. Understanding Attitudes and Predicting Social Behavior. Englewood Cliffs (NJ): Prentice-Hall; 1980.

52. Chudzickaczupała A, Grabowski D, Mello AL, et al. Application of the theory of planned behavior in academic cheating research-crosscultural comparison. Ethics Behav. 2016;26(8):638-659. doi:10.1080/ 10508422.2015.1112745 
53. Barton BK, Kologi SM, Siron A. Distracted pedestrians in crosswalks: an application of the theory of planned behavior. Transp Res Part F Psychol Behav. 2016;37:129-137. doi:10.1016/j. $\operatorname{trf} .2015 .12 .012$

54. Oluka OC, Sun Y, Komlan K, Sun L, Zhang L. Barriers to provision of lifestyle counseling to cancer survivors: a theory of planned behavior study. SAGE Open. 2017;7(2):1-9. doi:10.1177/2158244017709045

55. Koh H, Mackert M. A study exploring factors of decision to text while walking among college students based on Theory of Planned Behavior (TPB). J Am Coll Health. 2016;64(8):619-627. doi:10.1080/07448481.2016.1215986

56. Qin L, Kim Y, Tan X. Understanding the Intention of using mobile social networking apps across cultures. Int $J$ Human-Comput Interact. 2018;34(12):1183-1193. doi:10.1080/10447318.2018.1428262

57. Pelling EL, White KM. The theory of planned behavior applied to young people's use of social networking web sites. Cyberpsychol Behav. 2009;12(6):755-759.

58. Gopi M, Ramayah T. Applicability of theory of planned behavior in predicting intention to trade online. Int J Emerging Markets. 2007;2 (4):348-360. doi:10.1108/17468800710824509

59. Su D, Huang X. Research on online shopping intention of undergraduate consumer in China-based on the theory of planned behavior. Int Bus Res. 2011;4(1):86-92.

60. Knabe AP (2012). Applying Ajzen's theory of planned behavior to a study of online course adoption in public relations education [Doctoral dissertation]. doi:10.1094/PDIS-11-11-0999-PDN

61. Renzi, S. Differences in university teaching after learning management system adoption: an explanatory model based on Ajzen's theory of planned behavior (Unpublished doctorial dissertation). Perth:The University of Western Australia; 2011

62. Flack M, Morris M. Gambling-related beliefs and gambling behaviour: explaining gambling problems with the theory of planned behavior. Int $J$ Mental Health Addict. 2017;15(1):130-142. doi:10.1007/s11469-015-9611-9

63. Wood MD, Read JP, Mitchell RE, Brand NH. Do parents still matter? Parent and peer influences on alcohol involvement among recent high school graduates. Psychol Addict Behav. 2004;18(1):19-30. doi:10.1037/0893-164X.18.1.19

64. Chotpitayasunondh V, Douglas KM. How "phubbing" becomes the norm: the antecedents and consequences of snubbing via smartphone. Comput Human Behav. 2016;63:9-18. doi:10.1016/j. chb.2016.05.018

65. Lee SG. An integrative study of mobile technology adoption based on the technology acceptance model, theory of planned behavior and diffusion of innovation theory. Pers Soc Psychol Bull. 2003;29 (10):1328-1338.

66. Xu Y, Su SB, Lin DH. The relationship between parental factors, resistance efficacy and adolescents' new media dependence. Psychol Dev Educ. 2012;28(4):421-427.

67. Roberts JA, David ME. My life has become a major distraction from my cell phone: partner phubbing and relationship satisfaction among romantic partners. Comput Human Behav. 2016;54(C):134-141. doi:10.1016/j.chb.2015.07.058
68. Lu Y, Zhou T, Wang B. Exploring Chinese Users' Acceptance of Instant Messaging Using the Theory of Planned Behavior, the Technology Acceptance Model, and the Flow Theory. Elsevier Science Publishers B. V; 2009.

69. Li-tze H, Bentler PM. Cutoff criteria for fit indexes in covariance structure analysis: conventional criteria versus new alternatives. Struct Equ Modeling. 1999;6(1):1-55. doi:10.1080/10705519 909540118

70. Harman HH. Modern Factor Analysis. 3rd ed. London, England: The University of Chicago Press; 1976.

71. Ying XU. Adolescent new media dependence behavior: the roles of parental factors and refusal self-efficacy. Psychol Dev Educ. 2012;28:421-427. (Chinese).

72. Deng LY, Fang XY, Li YF, Wan JJ.Parental surveillance and juvenile problem behavior. Appl Psychol. 2006;12(4):305-311.

73. Park SK, Kim JY, Cho CB. Prevalence of internet addiction and correlations with family factors among South Korean adolescents. Adolescence. 2008;43(172):895-909.

74. Scholte R, Poelen E, Willemsen G, Boomsma D, Engels R. Relative risks of adolescent and young adult alcohol use: the role of drinking fathers, mothers, siblings and friends. Addict Behav. 2008;33 (1):1-16. doi:10.1016/j.addbeh.2007.04.015

75. Krohn MD, Skinner WF, Massey JL, Akers RL. Social learning theory and adolescent cigarette smoking: a longitudinal study. Soc Probl. 1985;32(5):455-473. doi:10.2307/800775

76. Kim HS. The mediating effects of self-identity in the influence of peer-alienation of youth to mobile phone dependency. $J$ Korea Contents Assoc. 2014;14(12):527-537.

77. Song MR, Kim SY. The relationship among peer attachment, ego-resilience and mobile phone dependency in middle school students. Forum Youth Culture. 2012;32:66-89.

78. Jiang K, Yang Z, Feng Z, Yu Z, Bao S, Huang Z. Mobile phone use while cycling: a study based on the theory of planned behavior. Transp Res Part F. 2019;64:388-400. doi:10.1016/j. trf.2019.05.020

79. Piazza AJ, Knowlden AP, Hibberd E, Leeper J, Paschal AM, Usdan S. Mobile device use while crossing the street: utilizing the theory of planned behavior. Accid Anal Prev. 2019;127:9-18. doi:10.1016/j.aap.2019.02.006

80. Sullman MJM, Hill T, Stephens AN. Predicting intentions to text and call while driving using the theory of planned behaviour. Transp Res Part F. 2018;58:405-413. doi:10.1016/j.trf.2018.05.002

81. Liao C, Chen JL, Yen DC. Theory of planning behavior (TPB) and customer satisfaction in the continued use of e-service: an integrated model. Comput Human Behav. 2007;23(6):2804-2822. doi:10.1016/j. chb.2006.05.006

82. Holland C, Rathod V. Influence of personal mobile phone ringing and usual intention to answer on driver error. Accid Anal Prev. 2013;50 (1):793-800. doi:10.1016/j.aap.2012.07.004

83. Fang J, Shao P. An extended TPB model to explain potential respondents' intention to participate in web-based surveys. In: International Conference on Wireless Communications. NETWORKING and Mobile Computing; 2007:6079-6082. 


\section{Publish your work in this journal}

Psychology Research and Behavior Management is an international, peer-reviewed, open access journal focusing on the science of psychology and its application in behavior management to develop improved outcomes in the clinical, educational, sports and business arenas. Specific topics covered in the journal include: Neuroscience, memory and decision making; Behavior modification and management; Clinical applications; Business and sports performance management; Social and developmental studies; Animal studies. The manuscript management system is completely online and includes a very quick and fair peer-review system, which is all easy to use. Visit http://www. dovepress.com/testimonials.php to read real quotes from published authors.

Submit your manuscript here: https://www.dovepress.com/psychology-research-and-behavior-management-journal 